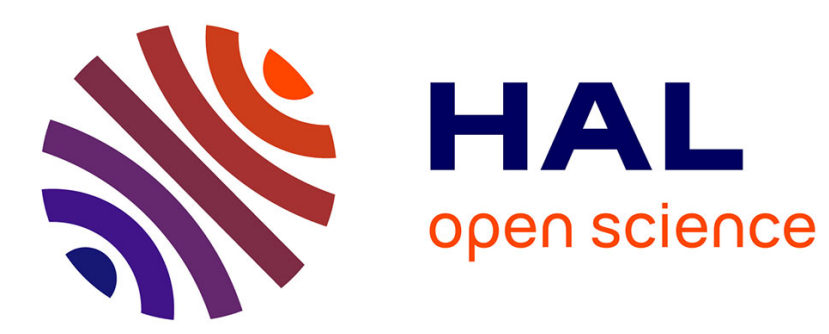

\title{
Strain-path dependent hardening models with rigorously identical predictions under monotonic loading
}

\author{
Yanfeng Yang, Gabriela Vincze, Cyrille Baudouin, Hocine Chalal, Tudor
}

Balan

\section{- To cite this version:}

Yanfeng Yang, Gabriela Vincze, Cyrille Baudouin, Hocine Chalal, Tudor Balan. Strain-path dependent hardening models with rigorously identical predictions under monotonic loading. Mechanics Research Communications, 2020, pp.103615. 10.1016/j.mechrescom.2020.103615 . hal-03071701

\section{HAL Id: hal-03071701 https://hal.science/hal-03071701}

Submitted on 16 Dec 2020

HAL is a multi-disciplinary open access archive for the deposit and dissemination of scientific research documents, whether they are published or not. The documents may come from teaching and research institutions in France or abroad, or from public or private research centers.
L'archive ouverte pluridisciplinaire HAL, est destinée au dépôt et à la diffusion de documents scientifiques de niveau recherche, publiés ou non, émanant des établissements d'enseignement et de recherche français ou étrangers, des laboratoires publics ou privés. 


\title{
Strain-path dependent hardening models with rigorously identical predictions under monotonic loading
}

\author{
Yanfeng YANG ${ }^{1}$, Gabriela VINCZE $^{2}$, Cyrille BAUDOUIN ${ }^{1}$, Hocine $_{\text {CHALAL }}^{3}$, Tudor BALAN ${ }^{*}$
}

\author{
${ }^{1}$ Arts et Metiers Institute of Technology, Université de Lorraine, LCFC, HESAM Université, F-57070 Metz, France \\ ${ }^{2}$ Center for Mechanical Technology and Automation, Department of Mechanical Engineering, University of Aveiro, Campus Universitário de Santiago, $3810-$ \\ 193, Portugal \\ ${ }^{3}$ Arts et Metiers Institute of Technology, Université de Lorraine, LEM3, HESAM Université, F-57070 Metz, France
}

*Corresponding author: Tudor Balan. Tel: +33.3.87.37.54.60; Email: tudor.balan@ensam.eu.

\begin{abstract}
Accurate sheet metal simulation often requires advanced strain-path dependent material models, in order to predict the material response under complex loading conditions, including monotonic, reverse and orthogonal paths. More and more flexible models imply higher and higher costs in terms of parameter identification, computer implementation and simulation time, and robust comparison is often compromised by the inconsistent predictions of advanced models under monotonic loading. In this paper, a simple and general approach is proposed for the alteration of advanced hardening models in order to make them rigorously identical to each other under monotonic loading. This objective was reached without any drawback other than the addition of the corresponding equations. On the contrary, the flexibility and accuracy of the selected models was improved, and the parameter identification procedure became simpler, more accurate and more robust. Three material models of increasing complexity were selected to demonstrate the interest of this approach with respect to a complete set of characterisation experiments for a DP600 sheet steel.
\end{abstract}

\section{Introduction}

The accuracy and reliability of sheet metal forming numerical simulation depends heavily on the finite element (FE) code ability to suitably describe the material behavior [1], [2]. The success of the numerical results relies on the quality of the adopted constitutive model [3]-[6], and on the identification of the material parameters [7].

During sheet metal forming processes, the material is generally subjected to complex loading conditions, including monotonic, reverse and orthogonal strain paths. The work hardening behaviour of the material becomes more complicated when the deformation path involves several steps [8]. In sheet metal forming simulation, the choice of hardening model has a strong influence for final prediction accuracy. The complexity of the material models generally means that their experimental identification requires a greater number of material characterization tests [9]. Regarding the mechanical characterization of sheet metal following complex deformation paths, the tests with strainpath change have a great importance because they highlight the kinematic hardening, linked to the Bauschinger effect and to the phenomena of stagnation of work hardening [10]-[12]. Taking these phenomena into account in the model leads to a better prediction of certain defects observed in the forming of the sheet metal [13]-[15].

In order to improve the consistency of hardening model comparison, the rigorous separation of the isotropic and kinematic components of the hardening model was attempted in the literature [11],[16]. The advantage of this modelling approach is that the model predictions under monotonic loading are independent of the kinematic hardening model and parameters. However, this approach could only be applied to the Armstrong-Frederick kinematic hardening model.

The objective of this work was to propose an alternative approach for the self-compensation of non-isotropic hardening terms in elasto-plastic constitutive models, in order to obtain identical predictions under monotonic loading and thus allow for consistent model comparison. The paper is structured as follows: The selected material, material characterization tests and corresponding results are shown in Section 2. Section 3 presents three hardening models of increasing complexity selected for comparison. Selfcompensated versions of these models are developed in Section 4, and their parameters are identified. Finally, the conclusion of this work is summarized in Section 5 .

\section{Material characterization}

A $1.2 \mathrm{~mm}$ thick DP600 AHSS sheet steel was used in this work. The aim of the selected characterization tests is to reveal the hardening behaviour of the materials under large monotonic deformations and during strain-path change. In particular, a reverse loading sequence was provided since this sequence frequently occurs in sheet metal forming processes. Rheological tests carried out consist of:

- UT - uniaxial tension in rolling direction (RD), transverse direction (TD) and diagonal direction (DD); 
- SS - simple shear in RD; RS - reversal shear in RD;

- OR - uniaxial tension followed by simple shear in RD.

Fig. 1 summarizes the main material characterization results for DP600. The tensile tests under different directions clearly illustrate the very weak plastic anisotropy of DP600, confirmed by the anisotropy coefficients $r_{0}=0.85$, $r_{45}=0.80$ and $r_{90}=1.0$. A very good experimental repeatability is also observed. The simple shear test revealed the material behavior at large deformation, as shear strains up to 0.7 could be reached. Reverse shear tests performed with shear pre-strain levels between 0.1 and 0.3 exhibit an important Bauschinger effect, fast transient behaviour with hardening stagnation and further resumption, under cumulated shear strain up to 1 . However, in uniaxial tension followed by simple shear test (OR), the cross-hardening effect was not significant. Therefore, the modeling of the orthogonal effect was not considered important in the current investigation.

\section{Constitutive framework and models}

The frame-invariance of the material model is insured by formulating the constitutive model in a convenient rotating frame, such that simple material time derivatives can be used. With this approach, classically adopted in most finite- element implementations of plasticity models, the material model is form-identical to a small-strain formulation, without loss of accuracy. During the plastic deformation process, the elasticity domain evolves in the stress space in a complex way: it can change its size, translate, rotate and even distort. Here, the shape of the yield surface is supposed fixed, while its size and center location may evolve according to an arbitrary hardening model. Given the isotropy of the tested material, and the paper's focus on hardening modeling, the von Mises yield surface is adopted throughout the paper.

The total strain rate tensor $\dot{\varepsilon}$ can be decomposed in elastic strain rate $\dot{\boldsymbol{\varepsilon}}^{e}$ and plastic strain rate $\dot{\boldsymbol{\varepsilon}}^{p}$. The hypo-elastic law expressed as

$\dot{\boldsymbol{\sigma}}=\boldsymbol{C}: \dot{\boldsymbol{\varepsilon}}^{e}=\boldsymbol{C}:\left(\dot{\boldsymbol{\varepsilon}}-\dot{\boldsymbol{\varepsilon}}^{p}\right)$

is considered, where $\boldsymbol{\sigma}$ is the Cauchy stress rate and $\boldsymbol{C}=$ $2 G \boldsymbol{I}_{4}^{\prime S}+K \boldsymbol{I} \otimes \boldsymbol{I}$ is the fourth-order tensor in isotropic linear elasticity. $G$ and $K$ can be calculated with using Young's modulus $E$ and Poisson's ratio $v$. The components of second order unit tensor $\boldsymbol{I}$ are the Kronecker deltas $\left(I_{k l}=\delta_{k l}\right)$. While $I_{4}^{\prime S}$ is the fourth-order symmetric deviatoric unit tensor and its components are $I_{4 i j k l}^{\prime S}=(1 / 2)\left(\delta_{i k} \delta_{j l}+\right.$
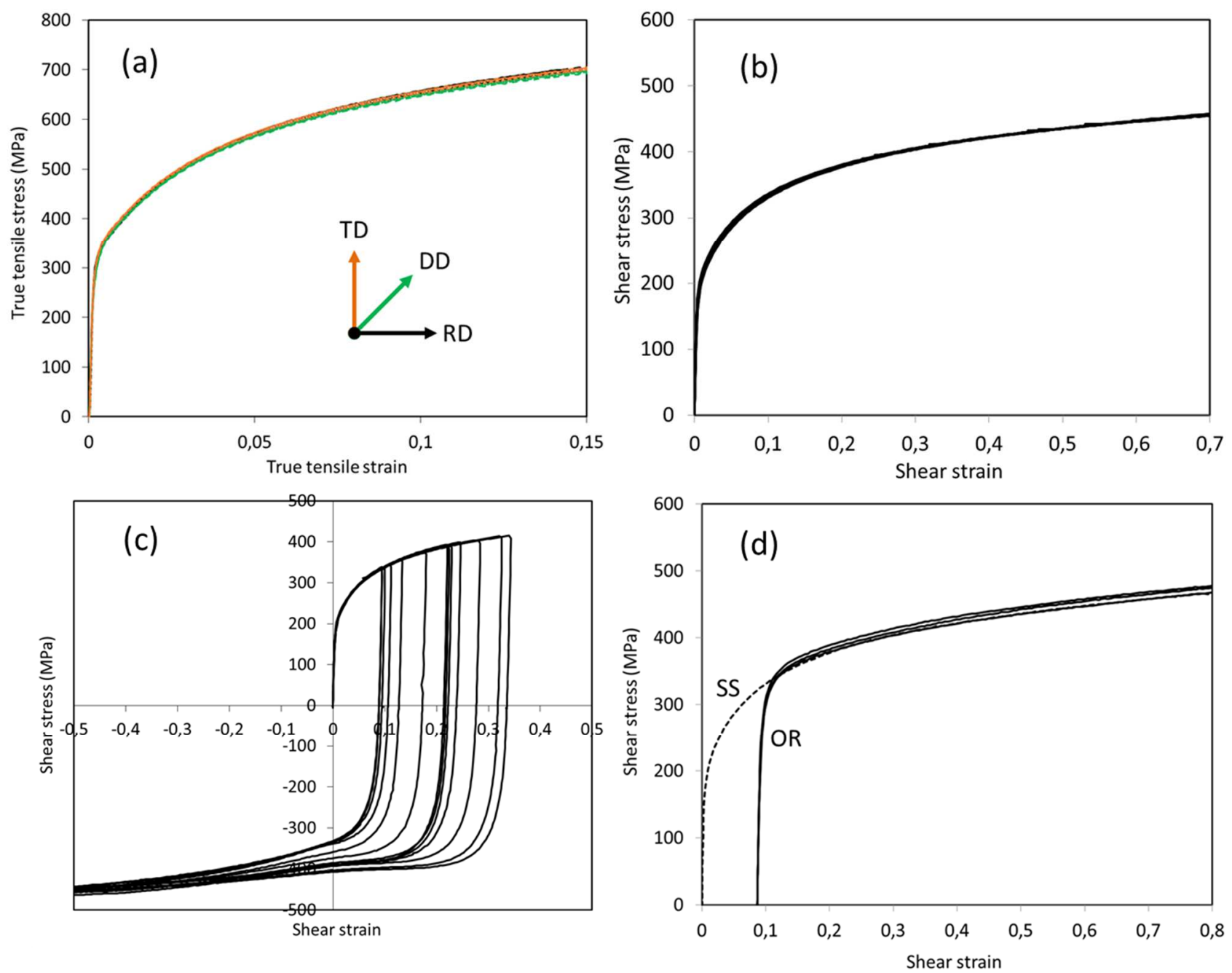

Fig. 1. Material characterization results of DP600: (a) uniaxial tension (UT) in RD (black curves), DD (green curves) and TD (orange curves); (b) simple shear (SS) in RD; (c) reverse shear (RS) in RD; (d) uniaxial tension followed by simple shear (OR) in RD. 
$\left.\delta_{i l} \delta_{j k}\right)-(1 / 3) \delta_{i j} \delta_{k l}$. The plastic strain rate tensor $\dot{\boldsymbol{\varepsilon}}^{p}$ is given by the associated flow rule

$\dot{\boldsymbol{\varepsilon}}^{p}=\dot{\lambda} \cdot \frac{\partial \bar{\sigma}}{\partial \boldsymbol{\sigma}}=\dot{\lambda} \cdot \boldsymbol{V}$

where $\boldsymbol{V}$ is the flow direction defined by the normality rule, $\bar{\sigma}\left(\boldsymbol{\sigma}^{\prime}-\boldsymbol{X}\right)$ is the equivalent stress using von Mises function and $\boldsymbol{\sigma}^{\prime}$ denotes the deviatoric part of the Cauchy stress tensor. The second order tensor $\boldsymbol{X}$ designates the backstress and $Y$ describes the size of the yield surface; their evolution is formally modeled by generic equations

$\dot{Y}=H_{Y} \cdot \dot{\lambda}$,

$\dot{\boldsymbol{X}}=\boldsymbol{H}_{\boldsymbol{X}} \cdot \dot{\lambda}$,

where $H_{Y}$ and $\boldsymbol{H}_{\boldsymbol{X}}$ represent isotropic hardening and kinematic hardening. The plastic multiplier $\dot{\lambda}$ is calculated with equation

$$
\dot{\lambda}=\frac{\boldsymbol{V}: \boldsymbol{C}: \dot{\boldsymbol{\varepsilon}}}{\boldsymbol{V}: \boldsymbol{C}: \boldsymbol{V}+\boldsymbol{V}: \boldsymbol{H}_{\boldsymbol{X}}+H_{Y}},
$$

deriving from the consistency condition. This generic modeling framework can be particularized to various yield functions, and various hardening models. According to the characterization tests of DP600, the hardening behavior of this material exhibits several specificities after reverse loading: early reyielding (Bauschinger effect), fast hardening followed by stagnation, and further hardening resumption at larger reverse strains. Consequently, this material was selected to investigate the ability of different types of hardening models to predict these features. In contrast, DP600 exhibits almost no anisotropy, and almost no crosshardening effect on the stress-strain curves; thus these features of plastic behavior will not be investigated.

More specifically, three representative hardening models were confronted, belonging to three categories widely encountered in the literature, with increasing levels of complexity: isotropic hardening, combined isotropickinematic hardening and a microstructure-based advanced hardening model.

\subsection{Swift-Voce isotropic hardening}

Generally, the size of the yield surface $Y$ is decomposed into an initial yield stress $Y_{0}$ and an isotropic hardening variable $R$,

$Y=Y_{0}+R$

The isotropic hardening models are the oldest, and the most commonly used in industry still nowadays. This type of model can only describe accurately monotonic loading, for example uniaxial tension (UT) or simple shear (SS). Thus the backstress is set to zero and the so-called Swift-Voce isotropic hardening model is adopted, which is a classical combination of a power law and a saturating law:
$\dot{R}_{v}=H_{R_{v}} \cdot \dot{\lambda}=C_{R} \cdot\left(R_{\text {sat }}-R_{v}\right) \cdot \dot{\lambda}$,

$\dot{R}_{S}=H_{R_{S}} \cdot \dot{\lambda}=n \cdot K^{1 / n}\left(K \varepsilon_{0}^{n}\right)^{1 / n} \cdot \dot{\lambda}$,

where $C_{R}$ and $R_{\text {sat }}$ are the parameters of Voce isotropic hardening described by variable $R_{v}$, and $K, \varepsilon_{0}, n$ are the parameters of Swift isotropic hardening $R_{s}$. The resulting isotropic hardening is given by

$\dot{Y}=\dot{R}=\dot{R}_{v}+\dot{R}_{s}$, with $Y_{0}=K \varepsilon_{0}^{n}$

$H_{Y}=H_{R_{v}}+H_{R_{S}}$.

The Swift-Voce combined law was used as it is repeatedly identified in the literature as one of the most adequate isotropic hardening law to describe large strain hardening behavior of sheet metals, in particular steels [12], [17].

\subsection{Chaboche combined hardening}

The so-called combined hardening models describe isotropic and kinematic hardening with two internal state variables $R$ and $\boldsymbol{X}$. The isotropic hardening $R$ uses the same equations (7)-(8) as in the previous model. Two backstress tensors are used to describe kinematic hardening with Armstrong-Frederick type equations,

$\dot{\boldsymbol{X}}_{\mathbf{1}}=\boldsymbol{H}_{X \mathbf{1}} \cdot \dot{\lambda}=C_{X 1} \cdot\left(X_{\text {sat } 1} \cdot \boldsymbol{n}-\boldsymbol{X}_{\mathbf{1}}\right) \cdot \dot{\lambda}$,

$\dot{\boldsymbol{X}}_{2}=\boldsymbol{H}_{X 2} \cdot \dot{\lambda}=C_{X 2} \cdot\left(X_{\text {sat } 2} \cdot \boldsymbol{n}-\boldsymbol{X}_{2}\right) \cdot \dot{\lambda}$,

where $C_{X 1}, X_{\text {sat } 1}, C_{X 2}, X_{\text {sat } 2}$ are material parameters. $\boldsymbol{n}=$ $\left(\boldsymbol{\sigma}^{\prime}-\boldsymbol{X}\right) / \bar{\sigma}$ designates the offset deviatoric stress direction. The rate of total kinematic hardening $\boldsymbol{X}$ is calculated with

$\dot{X}=\dot{X}_{1}+\dot{X}_{2}$

$H_{X}=H_{X 1}+H_{X 2}$

with initial values usually equal to zero (annealed material). The use of two backstress tensors is not compulsory, but is was shown to improve the predictions with respect to the classical Frederick-Armstrong model with only one term; this was also the case in this study. More than two backstress tensors are rarely proved necessary.

\subsection{Microstructure-based hardening}

On the basis of the microstructural evolution after twostage non-proportional loading at finite strains, a microstructural model was developed by Teodosiu and $\mathrm{Hu}$ [18]. This advanced hardening model is able to reproduce not only the Bauschinger effect but also other transient hardening phenomena during non-monotonic loading process. It is based on physical considerations, mainly the description of the evolution of the so-called planar persistent dislocation structures (PPDS) and their contribution to the 
hardening of the material. Proper description of PPDS evolution mechanisms reproduces the elementary transient phenomena observed on stress-strain evolution curves. This model involves four internal state variables: $R, \boldsymbol{X}, \boldsymbol{S}, \boldsymbol{P}$. The variable $R$ is a scalar, while $\boldsymbol{X}$ and $\boldsymbol{P}$ are second-order tensors and $\boldsymbol{S}$ is fourth order tensor.

The yield surface size is given as function of $R$ and $S$ by

$Y=Y_{0}+R+f|\boldsymbol{S}|$,

where $R$ describes the contribution of the randomly distributed dislocations to the isotropic hardening. The term $f|\boldsymbol{S}|$ represents the effect of PPDS on isotropic hardening, where $\boldsymbol{S}$ describes the directional strength of the PPDS and $f$ is a material parameter.

The kinematic hardening evolution law, described by the back-stress variable, is given by Eq. (11). Nevertheless, the saturation value $X_{\text {sat }}$ is no longer a material parameter in Teodosiu-Hu's model, but a function of the internal state variable $\boldsymbol{S}$. This dependency of $X_{\text {sat }}$ on the $\boldsymbol{S}$ variable is assumed of the form

$X_{\text {sat }}=X_{0}+(1-f)|S| \sqrt{r+(1-r) \beta_{S}^{2}}$,

where $X_{0}$ is the initial value of $X_{\text {sat }}$ and $r$ is a material parameter in Eq. (16). The ratio $\beta_{S}=S_{D} /|\boldsymbol{S}|$ is a measure of the change in the orientation of the current strain rate tensor with respect to the PPDS. This parameter is therefore considered to be an indicator of strain-path change. It evolves between 0 (orthogonal loading) and 1 (monotonic or reverse loading).

Experimental observations indicate that the PPDS associated with the current direction of the strain rate evolve quite differently from the rest of the PPDS during strain-path change. The variable $\boldsymbol{S}$ is therefore decomposed into two parts: $S_{D}$ (scalar) representing the strength associated with the currently active slip systems, and $S_{L}$ (fourth-order tensor), associated with the latent part of the PPDS. The decomposition of $\boldsymbol{S}$ takes the following form:

$S_{D}=N: S: N, S_{L}=S-S_{D} N \otimes N$,

where $\boldsymbol{N}=\dot{\boldsymbol{\varepsilon}}^{p} /\left|\dot{\boldsymbol{\varepsilon}}^{p}\right|$ represents the plastic strain rate direction. The evolution laws of $S_{D}$ and $S_{L}$ are given by

$\dot{S}_{D}=H_{S D} \cdot \dot{\lambda}=C_{S D}\left[g\left(S_{s a t}-S_{D}\right)-h S_{D}\right] \cdot \dot{\lambda}$,

where $S_{\text {sat }}$ and $C_{S D}$ govern the saturation value and evolution rate of $S_{D}$, and

$\dot{S}_{L}=\boldsymbol{H}_{S L} \cdot \dot{\lambda}=-C_{S L}\left(\frac{\left|\boldsymbol{S}_{L}\right|}{S_{\text {sat }}}\right)^{n_{L}} \boldsymbol{S}_{\boldsymbol{L}} \cdot \dot{\lambda}$,

where $C_{S L}$ and $n_{L}$ characterize the saturation rate of $\boldsymbol{S}_{L}$. The functions $g$ and $h$ in Eq. (18) have been introduced in order to further describe transient hardening after a change in strain-path. Their assumed mathematical forms are
$g=\left\{\begin{array}{c}1-\frac{C_{P}}{C_{S D}+C_{P}}, \text { if } \boldsymbol{P}: \boldsymbol{N} \geq 0 \\ (1+\boldsymbol{P}: \boldsymbol{N})^{n_{p}}\left(1-\frac{C_{P}}{C_{S D}+C_{P}} \cdot \frac{S_{D}}{S_{\text {sat }}}\right), \text { otherwise }\end{array}\right.$

and

$h=\frac{1}{2}\left(1-\frac{X: N}{X_{\text {sat }} \boldsymbol{n}: \boldsymbol{N}}\right)$.

In Eq. (20), $n_{p}$ is a material parameter and $\boldsymbol{P}$ is the internal state variable describing the polarity of the PPDS. Its evolution law is given by

$\dot{\boldsymbol{P}}=C_{P}(\boldsymbol{N}-\boldsymbol{P}) \cdot \dot{\lambda}$,

where $C_{P}$ characterizes the polarization rate of the PPDS in Eq. (22).

From Eqs. (17)-(19), one can obtain the time derivative of the norm of the $\boldsymbol{S}$ tensor as [13]

$|\dot{\boldsymbol{S}}|=H_{|\boldsymbol{S}|} \cdot \dot{\lambda}=\frac{1}{|\boldsymbol{S}|}\left[H_{S D} S_{D}-C_{S L}\left(\frac{\left|\boldsymbol{S}_{\boldsymbol{L}}\right|}{S_{s a t}}\right)^{n_{L}}\left|\boldsymbol{S}_{\boldsymbol{L}}\right|^{2}\right] \cdot \dot{\lambda}$,

where $|\boldsymbol{S}|=\sqrt{\left|\boldsymbol{S}_{\boldsymbol{L}}\right|^{2}+S_{D}^{2}}$. Thus, the scalar function $H_{Y}$ in Eq. (10) is deduced for this model as

$H_{Y}=H_{R}+f H_{|S|}$.

Remarkably, this advanced model reduces to the generic form assumed in Eqs (1)-(5) [13], [19], which simplifies its numerical implementation. Nevertheless, it involves a number of additional state variables, including second and fourth order tensors, interconnected through several rate and algebraic equations. It is used here as a representative candidate for advanced models that can reproduce complex transient behavior after strain path change (for example, hardening stagnation followed by hardening recovery at larger strains).

\subsection{Parameter identification of hardening models}

The material parameter identification is seen as an inverse problem. The gap between the experimental stress-strain curves and the simulated ones is quantified via an objective function $f_{o b j}$, which is minimized with respect to the material model's parameters [20], [21]. We are looking for $\min \left(f_{o b j}\right)$, with

$f_{o b j}=\sum_{i=1}^{N} \frac{1}{M_{i}} \sum_{j=1}^{M_{j}}\left(\frac{\sigma_{i, j}^{\text {exp }}-\sigma_{i, j}^{\text {sim }}}{\sigma_{i, j}^{\text {exp }}}\right)^{2}+\left(\frac{h_{i, j}^{\text {exp }}-h_{i, j}^{\text {sim }}}{h_{i, j}^{\text {exp }}}\right)$.

In order to improve the accuracy of the identification, a combined cost function is used [7], which considers the work hardening rate $h$ along with the stress values $\sigma . N$ is the number of tests, $M_{i}$ is the number of measurement points of the $i^{\text {th }}$ test. The upper indices 'sim' and 'exp' designate calculated values and experimental values, respectively. 
A Fortran program was developed to simulate the rheological tests (UT, SS, RS, OR) with the various models, independently of any commercial software. The optimization is performed with SciLab software, which provides an interactive call to the Fortran user program which allows data to be exchanged between SciLab and the Fortran program.

Fig. 2 shows simulation results of rheological tests (UT, SS and RS) by using the identified hardening parameters. It is clear that the selected isotropic hardening model can well predict monotonic loading test (UT, SS) up to large strains. However, this model failed to describe reverse shear because isotropic hardening model cannot capture the Bauschinger effect. The combined hardening is able to improve the predictions in reverse shear due to kinematic hardening. However, the accuracy of the combined hardening model is relatively smaller in UT and SS, compared with isotropic hardening. This inconsistency is a very serious drawback for material model comparison and selection. Finally, the microstructure-based hardening captured not only the Bauschinger effect in reversal tests, but also hardening stagnation and resumption at large deformation. Nevertheless, compared with isotropic hardening, this model also exhibits accuracy loss in monotonic loading (UT, SS). Obviously, this loss can be reduced by modifying the relative weight of monotonic tests in the cost function, yet it is not possible to rigorously eliminate it without seriously compromising the benefits observed under strain-path change. In the next section, a rigorous solution is proposed to eradicate this inconsistency.

\section{Self-compensated hardening models}

When only isotropic hardening is used to simulate a monotonic loading mode, say uniaxial tension, then variable $Y$ describes the evolution of the tensile stress component $\sigma_{T}$,

$$
Y \equiv \sigma_{T} \text { for isotropic hardening, }
$$

which is experimentally measured and directly used for the hardening model parameter identification. However, when kinematic hardening is added, this equality does not hold anymore: one obtains $\sigma_{T}=Y+X^{*}$, where $X^{*}$ is a scalar quantity corresponding to the tensile component of $\boldsymbol{X}$ under monotonic tensile loading [22]. This implies that any change to the model will impact the overall predictions under monotonic loading, even if the isotropic hardening part of the model is unchanged. An alternative to circumvent this drawback consists in explicitly modelling $\sigma_{T}$ and further calculating the size of the yield surface as
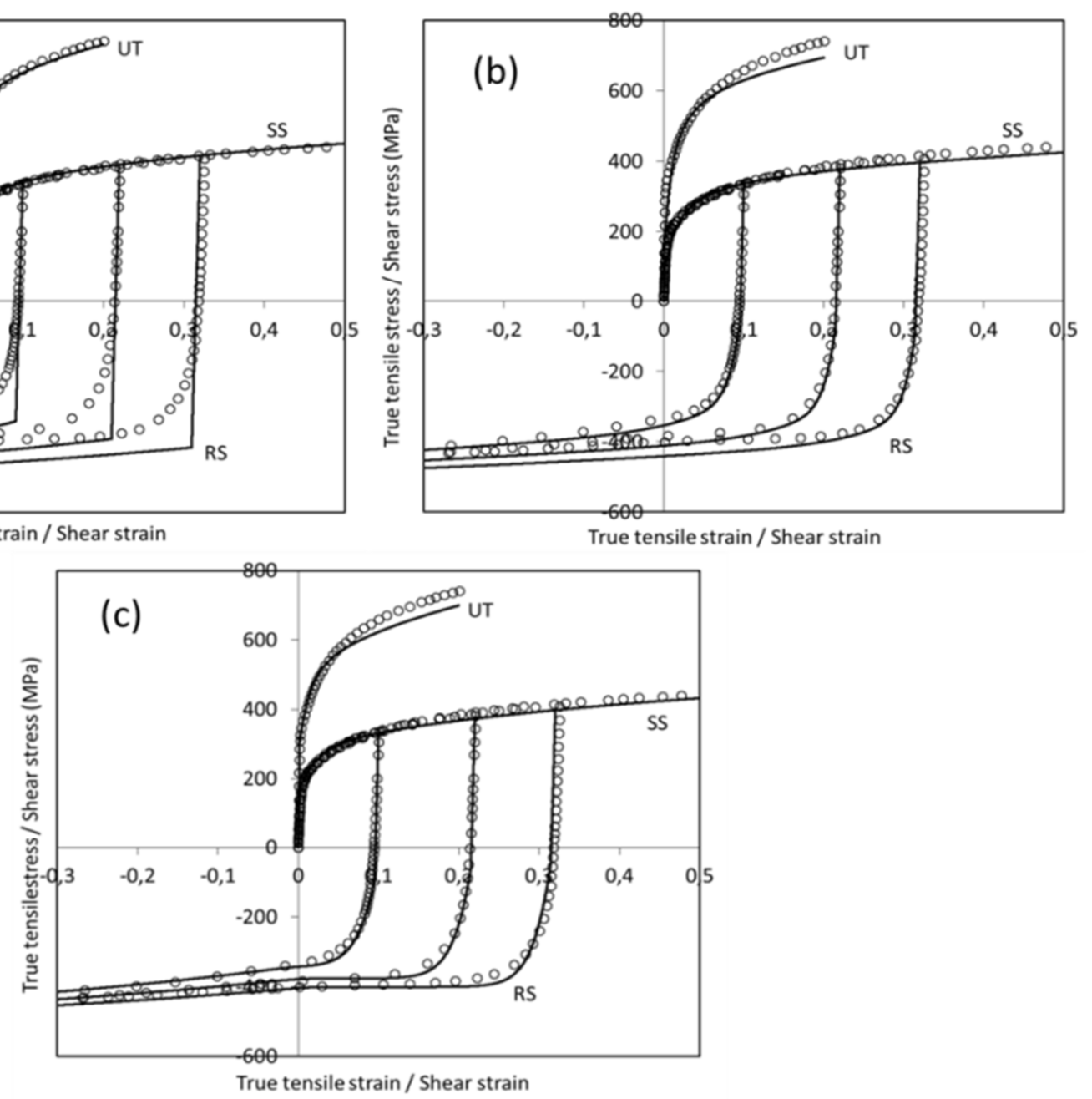

Fig. 2. Rheological predictions (solid line) of classical type hardening models: (a) Isotropic; (b) Combined; (c) Microstructural, and their comparisons of experimental results (symbols). 


$$
Y=\sigma_{T}-X^{*}
$$

This requires the explicit calculation of term $X^{*}$, which selfcompensates the contribution of kinematic hardening under monotonic loading. Several authors have derived and used its explicit expression for the particular case of ArmstrongFrederick kinematic hardening:

$$
X^{*}=X_{\text {sat }} \cdot\left(1-\exp \left(-C_{X} \cdot \bar{\varepsilon}^{p}\right)\right)
$$

where $\bar{\varepsilon}^{p}$ is the equivalent plastic strain.

However, the need for an algebraic expression of the selfcompensating term prevented the extension of this approach to more complex models, so this approach was only applied to the Armostrong-Frederick model in the literature. In this paper, the self-compensating terms are determined using rate equations, which provides a much more versatile approach.

\subsection{Self-compensated combined hardening}

In the proposed approach, the isotropic part of the hardening model describes the apparent flow stress under monotonic loading $\sigma_{T}$ by the Swift-Voce rate equation,

$\dot{\sigma}_{T}=H_{\sigma_{T}} \cdot \dot{\lambda}, H_{\sigma_{T}}=H_{R_{v}}+H_{R_{S}}$

The size $Y$ of the yield surface is determined by Eq. (27), where $X^{*}$ is also described by rate equations, corresponding to the tensile component of $\boldsymbol{X}$ under monotonic tensile loading:

$\dot{X}_{1}=H_{X 1} \cdot \dot{\lambda}, H_{X 1}=C_{X 1}\left(X_{\text {sat } 1}-X_{1}\right)$,

$\dot{X}_{2}=H_{X 2} \cdot \dot{\lambda}, H_{X 2}=C_{X 2}\left(X_{s a t 2}-X_{2}\right)$,

$\dot{X}^{*}=\dot{X}_{1}+\dot{X}_{2}$,

$H_{X}^{*}=H_{X 1}+H_{X 2}$.

The rate form of Eq. (27) writes:

$H_{Y}=H_{\sigma_{T}}-H_{X}^{*}$

In this alternative method, the monotonic flow curve is described independently of the kinematic hardening model and its parameters, which makes it more robust and especially convenient for model comparison. Eqns. (30)-(33) are directly inspired from the Eqns. (11)-(14) of the kinematic hardening model. Although it gives the same results as the closed-form approach (28), this approach based on rate equations is much more suitable for generalization to more complex hardening models.

\subsection{Self-compensated microstructure-based hardening model}

A self-compensated version of the Teodosiu-Hu model is introduced here for the first time. Again, $\sigma_{T}$ is governed by Eq. (29) and the size $Y$ of the yield surface is determined by

$Y=\sigma_{T}-\sigma^{*}$

where $\sigma^{*}$ is a scalar variable whose evolution equation directly derives from Eqn. (15):

$\sigma^{*}=X^{*}+f S_{D}^{*}$,

$\dot{X}^{*}=H_{X}^{*} \cdot \dot{\lambda}, H_{X}^{*}=C_{X}\left(X_{s a t}-X^{*}\right)$,

and $S_{D}^{*}$ is a scalar determined by

$\dot{S}_{D}^{*}=H_{S D}^{*} \cdot \dot{\lambda}=C_{S D}\left[g^{*}\left(S_{s a t}-S_{D}^{*}\right)-h^{*} S_{D}^{*}\right] \cdot \dot{\lambda}$.

Functions $h^{*}$ and $g^{*}$ write:

$h^{*}=0.5 \cdot\left(1-\frac{X^{*}}{X_{\text {sat }}}\right)$,

$g^{*}=1-\frac{C_{P}}{C_{S D}+C_{P}}\left|\frac{S_{D}^{*}}{S_{\text {sat }}}-P^{*}\right|$,

and $P^{*}$ is a scalar variable following

$\dot{P}^{*}=H_{P}^{*} \cdot \dot{\lambda}, H_{P}^{*}=C_{P}\left(1-P^{*}\right)$.

The rate form of Eq. (35) can be rewritten as

$H_{Y}=H_{\sigma_{T}}-H_{\sigma}^{*}, H_{\sigma}^{*}=H_{X}^{*}+f H_{S D}^{*}$.

Of course, the core of the Teodosiu-Hu model - Eqns. (15)(22) - remains unchanged.

Compared with the classical version of the microstructure-based hardening model, the selfcompensated version's predictions under monotonic loading are rigorously identical to the selected isotropic hardening model, without being affected by any further change in the parameters of kinematic hardening and cross hardening of this model. In addition to this significant improvement in consistency, it also brings some advantages in terms of parameter identification. The isotropic part of the hardening model can be identified by using only the available monotonic tests, and then its parameters can be fixed once and for all, since the monotonic simulations will be strictly identical. Therefore, any isotropic model can be adopted and identified independently. Then, in the second step, only the rest of the parameters need to be determined with respect only to the remaining experiments, without any loss in accuracy. 


\subsection{Parameter identification of self-compensated hardening models}

The two self-compensated hardening models discussed above were implemented in the developed Fortran program and their parameters were identified with the help of the identification method presented in Section 3.4, and the twostep procedure discussed above. The identified parameters of all models, classical and self-compensated, are summarized in Table 1. As expected, the first six parameters of the selfcompensated models are identical to those of the isotropic Swift-Vice (SV) model.

Table 1. Identified parameters for the classical and selfcompensated hardening models for DP600.

\begin{tabular}{cccccc}
\hline & \multicolumn{3}{c}{ Classical } & \multicolumn{2}{c}{ Self-compensated } \\
\cline { 2 - 6 } & S-V & Chaboche & Teodosiu & Chaboche & Teodosiu \\
\hline$C_{R}$ & 44.8 & 62 & 30.8 & \multicolumn{2}{c}{44.8} \\
$R_{\text {sat }}(\mathrm{MPa})$ & 136.9 & 84 & 93 & \multicolumn{2}{c}{136.9} \\
$K(\mathrm{MPa})$ & 664.1 & 449 & - & 664.1 \\
$n$ & 0.32 & 0.5 & - & \multicolumn{2}{c}{0.32} \\
$\varepsilon_{0}$ & 0.004 & 0.001 & - & \multicolumn{2}{c}{0.004} \\
$C_{X 1}$ & - & 157.5 & - & 90.2 & - \\
$X_{\text {sat } 1}(\mathrm{MPa})$ & - & 184 & - & 198.4 & - \\
$C_{X 2}$ & - & 28.7 & - & 5.9 & - \\
$X_{\text {sat } 2}(\mathrm{MPa})$ & - & 79 & - & 124.7 & - \\
$C_{X}$ & - & - & 65.1 & - & 65.1 \\
$X_{0}(\mathrm{MPa})$ & - & - & 168.2 & - & 168.2 \\
$S_{S a t}(\mathrm{MPa})$ & - & - & 405.3 & - & 565.6 \\
$C_{S D}$ & - & - & 2.66 & - & 2.85 \\
$C_{S L}$ & - & - & 1.2 & - & 1.2 \\
$C_{P}$ & - & - & 0.58 & - & 0.67 \\
$n_{L}$ & - & - & 0 & - & 0 \\
$n_{p}$ & - & - & 609.4 & - & 890 \\
$f$ & - & - & 0.04 & - & 0.79 \\
$r$ & - & - & 0.8 & - & 0.8 \\
\hline & & & & &
\end{tabular}

Fig. 3 shows the rheological test predictions by using the two self-compensated hardening models. Comparison to the isotropic hardening predictions in Fig. 2(a) shows that both self-compensated hardening models predict identical responses in monotonic loading. This improvement came with no degradation of the reverse loading predictions, which on the contrary could improve due to the rigorous separation of the parameter identification sequences. In particular, the Teodosiu-Hu model almost perfectly reproduces the entire set of experimental curves. Even the self-compensated Chaboche model provides excellent predictions of reverse shear for moderate pre-strains values - up to 0.1 . Furthermore, any isotropic hardening can also be adopted in place of the Swift-Voce one, without impact on the nonmonotonic predictions: the two parameter identification steps are truly independent of each other.
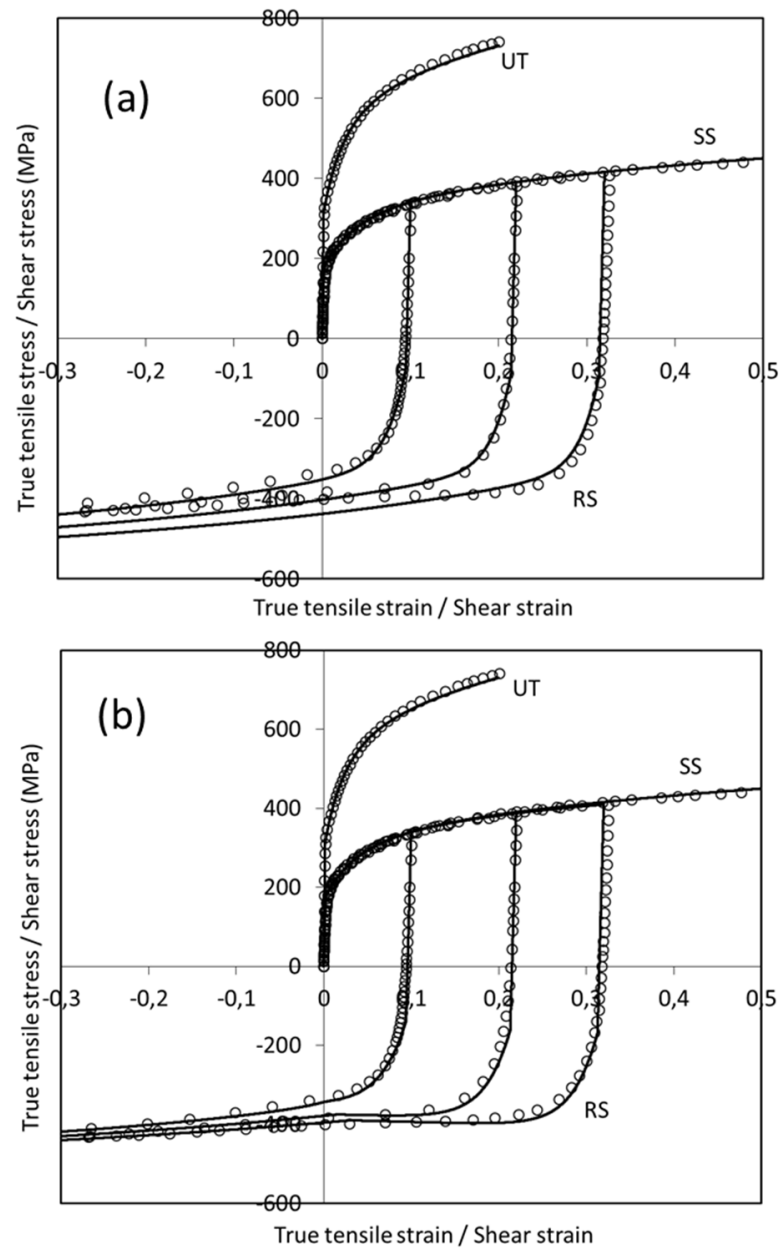

Fig. 3. Rheological predictions (solid line) of selfcompensated type hardening models: (a) Combined; (b) Microstructural; symbols designate experimental results.

\section{Conclusions}

In this paper, a novel approach was applied to make hardening models of various types identical under monotonic loading conditions. The proposed approach is more general than former closed-form attempts, and could be extended to an advanced microstructure-based hardening model. The resulting models have several advantages:

- Their predictions are rigorously identical to the (arbitrarily) selected isotropic hardening model;

- Their parameter identification is completely split in two independent sequences, one for the isotropic hardening part and one for the remaining parameters, without interference;

- The isotropic hardening part of each model can be freely selected and further modified, independently of the rest of the model;

- The self-compensated version of any model can be implemented in FE codes without additional programing: only the isotropic part of the hardening model is concerned, which can be simply implemented in tabular form. Thus, any available model can be "compensated" non-intrusively.

Three categories of classical and self-compensated models were selected, from simple isotropic hardening to 
advanced microstructure-based hardening. Their parameters were identified with respect to an extended set of monotonic and sequential characterization tests up to cumulated strains of 1 . The superior robustness of the self-compensated models was clearly demonstrated in terms of simultaneously improved consistency, accuracy and robustness. In their selfcompensated versions, the three models exhibit a clear progression in accuracy from isotropic to combined and finally to "advanced" hardening models, with no inconsistency. This approach provides a convenient ground for the subsequent material model comparison and selection with respect to specific applications. Future work will explore the relative accuracy of the proposed models and parameter sets in springback predictions for sheet metal forming applications.

\section{Acknowledgements}

The first author is grateful to the China Scholarship Council for providing him a $\mathrm{PhD}$ grant during the preparation of this work. GV acknowledges support from COMPETE 2020, in its FEDER/FNR component and the Portuguese Foundation of Science and Technology (FCT), in its State Budget component (OE) through projects POCI-01-0145FEDER-032466. The authors thank Dr Leopold Wagner from voestalpine Stahl $\mathrm{GmbH}$, Austria for fruitful discussions and for providing the DP600 material.

\section{References}

[1] P. Hu, D. Zhou, and J. Lian, "A new modified algorithm for elasticplastic FEM: Quasi-chord modulus method," Mech. Res. Commun., vol. 17, no. 6, pp. 365-374, 1990.

[2] P. Flores et al., "Model identification and FE simulations: effect of different yield loci and hardening laws in sheet forming," Int. J. Plast., vol. 23, no. 3, pp. 420-449, 2007.

[3] F. Yoshida and T. Uemori, "A model of large-strain cyclic plasticity describing the Bauschinger effect and workhardening stagnation," Int. J. Plast., vol. 18, no. 5-6, pp. 661-686, 2002.

[4] E. Rauch, J. Gracio, F. Barlat, and G. Vincze, "Modelling the plastic behaviour of metals under complex loading conditions," Model. Simul. Mater. Sci. Eng., vol. 19, no. 3, p. 035009, 2011.

[5] F. Barlat, G. Vincze, J. Grácio, M.-G. Lee, E. Rauch, and C. Tomé, "Enhancements of homogenous anisotropic hardening model and application to mild and dual-phase steels," Int. J. Plast., vol. 58, pp. 201-218, 2014.

[6] T. Clausmeyer, A. Güner, A. E. Tekkaya, V. Levkovitch, and B. Svendsen, "Modeling and finite element simulation of loading-pathdependent hardening in sheet metals during forming," Int. J. Plast., vol. 63, pp. 64-93, 2014.

[7] H. Haddadi, S. Bouvier, M. Banu, C. Maier, and C. Teodosiu, "Towards an accurate description of the anisotropic behaviour of sheet metals under large plastic deformations: modelling, numerical analysis and identification," Int. J. Plast., vol. 22, no. 12, pp. 2226-2271, 2006.

[8] M. Bambach, H. M. Bücker, S. Heppner, M. Herty, and I. N. Vladimirov, "Characteristics of testing conditions for constitutive models in metal plasticity," J. Eng. Math., vol. 88, no. 1, pp. 99-119, 2014.

[9] S. Bouvier, B. Gardey, H. Haddadi, and C. Teodosiu, "Characterization of the strain-induced plastic anisotropy of rolled sheets by using sequences of simple shear and uniaxial tensile tests," J. Mater. Process. Technol., vol. 174, no. 1-3, pp. 115-126, 2006.

[10]J. Wang, V. Levkovitch, F. Reusch, B. Svendsen, J. Huetink, and M. Van Riel, "On the modeling of hardening in metals during nonproportional loading," Int. J. Plast., vol. 24, no. 6, pp. 1039-1070, 2008.

[11] J. Carbonniere, S. Thuillier, F. Sabourin, M. Brunet, and P.-Y. Manach, "Comparison of the work hardening of metallic sheets in bendingunbending and simple shear," Int. J. Mech. Sci., vol. 51, no. 2, pp. 122-
$130,2009$.

[12]L. Sun and R. Wagoner, "Proportional and non-proportional hardening behavior of dual-phase steels," Int. J. Plast., vol. 45, pp. 174-187, 2013.

[13]B. Haddag, T. Balan, and F. Abed-Meraim, "Investigation of advanced strain-path dependent material models for sheet metal forming simulations," Int. J. Plast., vol. 23, no. 6, pp. 951-979, 2007.

[14]J. Liao, J. A. Sousa, A. B. Lopes, X. Xue, F. Barlat, and A. B. Pereira, "Mechanical, microstructural behaviour and modelling of dual phase steels under complex deformation paths," Int. J. Plast., vol. 93, pp. 269290, 2017.

[15] J. Qin, B. Holmedal, K. Zhang, and O. S. Hopperstad, "Modeling strainpath changes in aluminum and steel," Int. J. Solids Struct., vol. 117, pp. 123-136, 2017.

[16] B. Chun, J. Jinn, and J. Lee, "Modeling the Bauschinger effect for sheet metals, part I: theory," Int. J. Plast., vol. 18, no. 5-6, pp. 571-595, 2002.

[17]G. Venet, T. Balan, C. Baudouin, and R. Bigot, "Direct usage of the wire drawing process for large strain parameter identification," Int. J. Mater. Form., vol. 12, no. 5, pp. 875-888, 2019.

[18]C. Teodosiu and Z. Hu, "Evolution of the intragranular microstructure at moderate and large strains: modelling and computational significance," Simul. Mater. Process. Theory Methods Appl., pp. 173$182,1995$.

[19]T. Balan, "On the numerical implementation of elasto-plastic constitutive equations for metal forming," Romanian J. Tech. Sci.-Appl. Mech., vol. 60, no. 1-2, pp. 89-104, 2015.

[20] B. Chaparro, S. Thuillier, L. Menezes, P.-Y. Manach, and J. Fernandes, "Material parameters identification: Gradient-based, genetic and hybrid optimization algorithms," Comput. Mater. Sci., vol. 44, no. 2, pp. 339 346, 2008.

[21] A. Andrade-Campos, R. De-Carvalho, and R. Valente, "Novel criteria for determination of material model parameters," Int. J. Mech. Sci., vol. 54, no. 1, pp. 294-305, 2012.

[22] Y. Yang and T. Balan, "Prediction of the yield surface evolution and some apparent non-normality effects after abrupt strain-path change using classical plasticity," Int. J. Plast., vol. 119, pp. 331-343, 2019. 